\title{
Universiteit
}

Leiden

The Netherlands

\section{Statistical fluxes and the Curie-Weiss metal state}

Wu, K.; Weng, Z.Y.; Zaanen, J.

\section{Citation}

Wu, K., Weng, Z. Y., \& Zaanen, J. (2011). Statistical fluxes and the Curie-Weiss metal state. Physical Review B, 84(11), 113113. doi:10.1103/PhysRevB.84.113113

Version: $\quad$ Not Applicable (or Unknown)

License: $\quad$ Leiden University Non-exclusive license

Downloaded from: https://hdl.handle.net/1887/61311

Note: To cite this publication please use the final published version (if applicable). 


\title{
Statistical fluxes and the Curie-Weiss metal state
}

\author{
Kai Wu, ${ }^{1}$ Zheng-Yu Weng, ${ }^{1}$ and Jan Zaanen ${ }^{2}$ \\ ${ }^{1}$ Institute for Advanced Study, Tsinghua University, Beijing, 100084, China \\ ${ }^{2}$ Instituut Lorentz for Theoretical Physics, Leiden University, Leiden, P.O. Box 9506, 2300 RA Leiden, The Netherlands
}

(Received 5 September 2011; published 30 September 2011)

\begin{abstract}
We predict a new state of matter in the triangular $t-J$ model in a high doping regime. Due to the altered role of quantum statistics the spins are "localized" in statistical Landau orbits, while the charge carriers form a Bose metal that feels the spins through random gauge fields. In contrast to the Fermi-liquid state, this state naturally exhibits a Curie-Weiss susceptibility, large thermopower, and linear-temperature resistivity, explaining the physics of $\mathrm{Na}_{x} \mathrm{CoO}_{2}$ at $x>0.5$. A "smoking gun" prediction for neutron scattering is presented.
\end{abstract}

DOI: 10.1103/PhysRevB.84.113113

Introduction. Among the great challenges in quantum matter physics is the question whether Mottness-the drastic change of Hilbert space structure due to strong local interactions-might form the condition for non-Fermi liquid states of fermionic matter to occur. ${ }^{1,2}$ Among others, this is the point of departure of Anderson's resonating valence bond (RVB) proposal for high- $T_{c}$ superconductivity. ${ }^{3}$ At the electron densities in the proximity of the half-filled Mott insulator, the Mott projections are most evident and it is much less evident whether they matter at all at very high (or dilute) electron densities. Resting on a representation ${ }^{2,4}$ that makes explicit the altered nature of the quantum statistics due to Mottness, we demonstrate here the existence of an internally consistent, stable saddle point at high dopings on a triangular lattice. This is a spin-charge separated state where the doublons (carrying charge) and spinons (carrying spin) are coded as hard core bosons that communicate with each other via statistical fluxes. The surprise is in the spin sector: the statistical gauge fields act like a uniform magnetic field causing Landau quantization of the spinon states. The spinons are localized in Landau orbits of the lowest Landau level (LLL) at low temperatures, with the effect that these behave as free Curie-Weiss spins with large entropy. The doublons get in turn scattered by the random gauge fluxes associated with the localized spinons, similarly as in gauge glass models.

This might sound far fetched. However, the microscopy matches that of the highly overdoped $\mathrm{Na}_{x} \mathrm{CoO}_{2}$ system ${ }^{5-7}$ with $x>0.5$. A very strange metal is formed in this cobaltate, characterized by a high density of free Curie-Weiss spins that "appear out of the blue" while it is a rather good hole-type thermoelectric material ${ }^{8}$ given its large thermopower combined with a relatively low resistivity. We will show here that the magnetic (Fig. 1), thermoelectric (Fig. 2), and resistive (Fig. 3) properties of the cobaltate are consistent with the state described in the previous paragraph. We notice that earlier attempts ${ }^{9-14}$ also have potentially explained the aforementioned Curie-Weiss metal behavior of the cobaltates by invoking a very small bandwidth or strong disorder localization, however we present a smoking gun prediction that can be straightforwardly tested by experiment: the spin excitation spectrum should carry the fingerprints of the "spontaneous" Landau quantization (Fig. 4).

Similar to the layered structure in the high- $T_{c}$ cuprates, the $\mathrm{CoO}_{2}$ layer is believed to play an essential role in determining the low-energy physics in the $\mathrm{Na}_{x} \mathrm{CoO}_{2}$ compound. With
PACS number(s): 71.10.Hf, 71.27.+a, 72.80.Ga

electrons doping introduced by $\mathrm{Na}$, the electron hopping at the partially filled $t_{2 g}$ orbitals of the $\mathrm{Co}^{+3}$ ions and spin correlations between $\mathrm{Co}^{+4}$ ions in a $\mathrm{CoO}_{2}$ layer may be minimally described by the $t-J$ model ${ }^{15-17}$ on a triangular lattice with hopping amplitude $t<0 .{ }^{18-20}$ Here the Hilbert space is constrained by $\sum_{\sigma} c_{i \sigma}^{\dagger} c_{i \sigma} \geqslant 1$, that is, each lattice site is either singly occupied by an electron $\left(\mathrm{Co}^{+4}\right)$ or doubly occupied by electrons $\left(\mathrm{Co}^{+3}\right)$ without allowing the empty site $\left(\mathrm{Co}^{+5}\right)$. What we will be interested in is the highly overdoped regime of this model, where the RVB ${ }^{15-17}$ correlations induced by the superexchange coupling disappear and the hopping processes of both charge and spin become dominant.

Sign structure. To identify the new saddle point, we have to rely on a particular representation that is superficially reminiscent of the standard slave bosons but is actually quite different. This "phase string" representation" ${ }^{2,4}$ is making explicit the non-Fermi-Dirac nature of the quantum statistics in doped Mott insulators. According to this formalism, the electron operator $c_{i \sigma}$ can be fully "bosonized" by $c_{i \sigma}^{\dagger}=d_{i}^{\dagger} b_{i-\sigma} e^{-i \Theta_{i \sigma}^{\text {string }}}$, where the doublon and spinon creation operators $d_{i}^{\dagger}$ and $b_{i \sigma}^{\dagger}$ are both bosonic, which satisfy an equality (Mott) constraint $n_{l}^{d}+\sum_{\sigma} n_{l \sigma}^{b}=1$ with $n_{l}^{d}$ and $n_{l \sigma}^{b}$ denoting the doublon and spinon occupation numbers, respectively. Here the fermionic commutations of the electron operators are ensured by the topological phase $\Theta_{i \sigma}^{\text {string }} \equiv\left[\Phi_{i}^{s}+\sigma \Phi_{i}^{d}\right] / 2$, with $\Phi_{i}^{s} \equiv \sum_{l \neq i} \theta_{i}(l) \sum_{\sigma} \sigma n_{l \sigma}^{b}$ and $\Phi_{i}^{d} \equiv \sum_{l \neq i} \theta_{i}(l)\left(1-n_{l}^{d}\right)$,where $\theta_{i}(l)=\operatorname{Im}\left(z_{l}-z_{i}\right)\left(z_{i}\right.$ is the complex coordinate of site $i$ ). In this representation, the hopping and superexchange terms of the $t-J$ model become

$$
\begin{gathered}
H_{t}=t \sum_{\langle i j\rangle \sigma} \hat{D}_{j i} \hat{B}_{i j}^{\sigma}+\text { H.c. } \\
H_{J}=-\frac{J}{2} \sum_{\langle i j\rangle \sigma} \hat{B}_{i j}^{\sigma} \hat{B}_{j i}^{-\sigma}-\frac{J}{2} \sum_{\langle i j\rangle \sigma} n_{i \sigma}^{b} n_{j-\sigma}^{b},
\end{gathered}
$$

in terms of $\hat{D}_{i j} \equiv e^{i A_{i j}^{s}} d_{i}^{\dagger} d_{j}$ and $\hat{B}_{i j}^{\sigma} \equiv e^{-i \sigma A_{i j}^{d}} b_{i \sigma}^{\dagger} b_{j \sigma}$. In this way, the remnant sign structure after Mott projection has been made explicit while it is precisely represented by the topological link variables $A_{i j}^{s}$ and $A_{i j}^{d}$. Obviously there would 


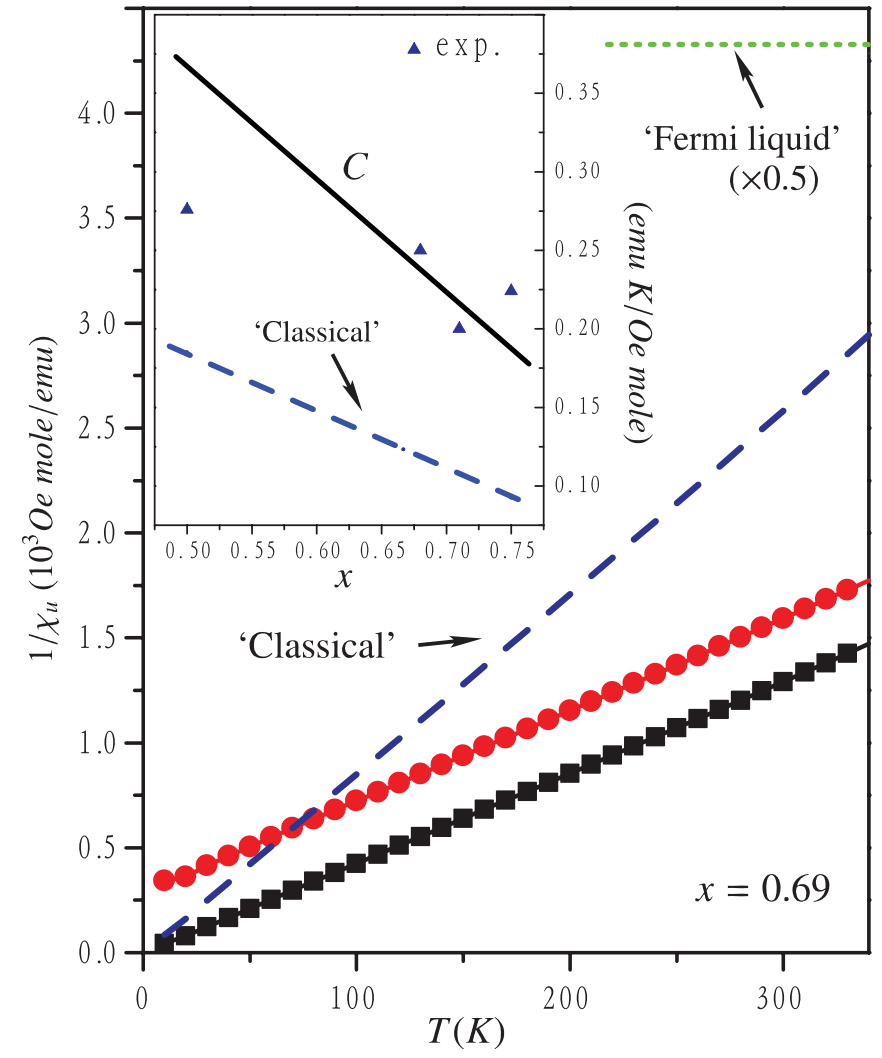

FIG. 1. (Color online) The spin part of the present electron fractionalization state exhibits a Curie $T$-dependent uniform susceptibility $\chi_{u}=C / T$ (full squares) at $x=0.69$. The Weiss term in $\chi_{u}$ appears as an RPA correction (full circles). The Fermi liquid (dotted) and classical limit (dashed) results are also shown for comparison. Inset: the coefficient $C$ (solid) and the classical limit $C_{\mathrm{cl}}$ (dashed) vs doping $x$, in which the experimental data are marked by full triangles. ${ }^{7,22,23}$

be no "sign problem" should $A_{i j}^{s}=A_{i j}^{d}=0$ in such a fully bosonized model with $t<0$. Here $A_{i j}^{s}$ and $A_{i j}^{d}$ satisfy

$$
\begin{aligned}
& \sum_{\langle i j\rangle \in \partial S} A_{i j}^{S}=\pi \sum_{l \in S} \sum_{\sigma} \sigma n_{l \sigma}^{b}, \\
& \sum_{\langle i j\rangle \in \partial S} A_{i j}^{d}=\pi \sum_{l \in S}\left(1-n_{l}^{d}\right),
\end{aligned}
$$

where $\partial S$ denotes the boundary of an area $S$.

Electron fractionalization saddle point. The precise sign structure identified above will be crucial in constructing the following saddle point which respects the gauge invariance associated with $A_{i j}^{s}$ and $A_{i j}^{d}$, together with time reversal and spin rotational symmetries. Since the RVB pairing is irrelevant at high doping, the gauge-invariant $\hat{D}_{i j}$ and $\hat{B}_{i j}^{\sigma}$ in Eqs. (1) and (2) will be natural order parameters, resulting in an effective $H_{\text {eff }}=H_{d}+H_{s}$ :

$$
\begin{gathered}
H_{d}=-t_{d} \sum_{\langle i j\rangle} e^{-i a_{i j}}\left(e^{i A_{i j}^{s}} d_{i}^{\dagger} d_{j}\right)+\text { H.c. } \\
H_{s}=-t_{s} \sum_{\langle i j\rangle \sigma} e^{-i a_{i j}}\left(e^{-i \sigma A_{i j}^{d}} b_{i \sigma}^{\dagger} b_{j \sigma}\right)+\text { H.c. }
\end{gathered}
$$

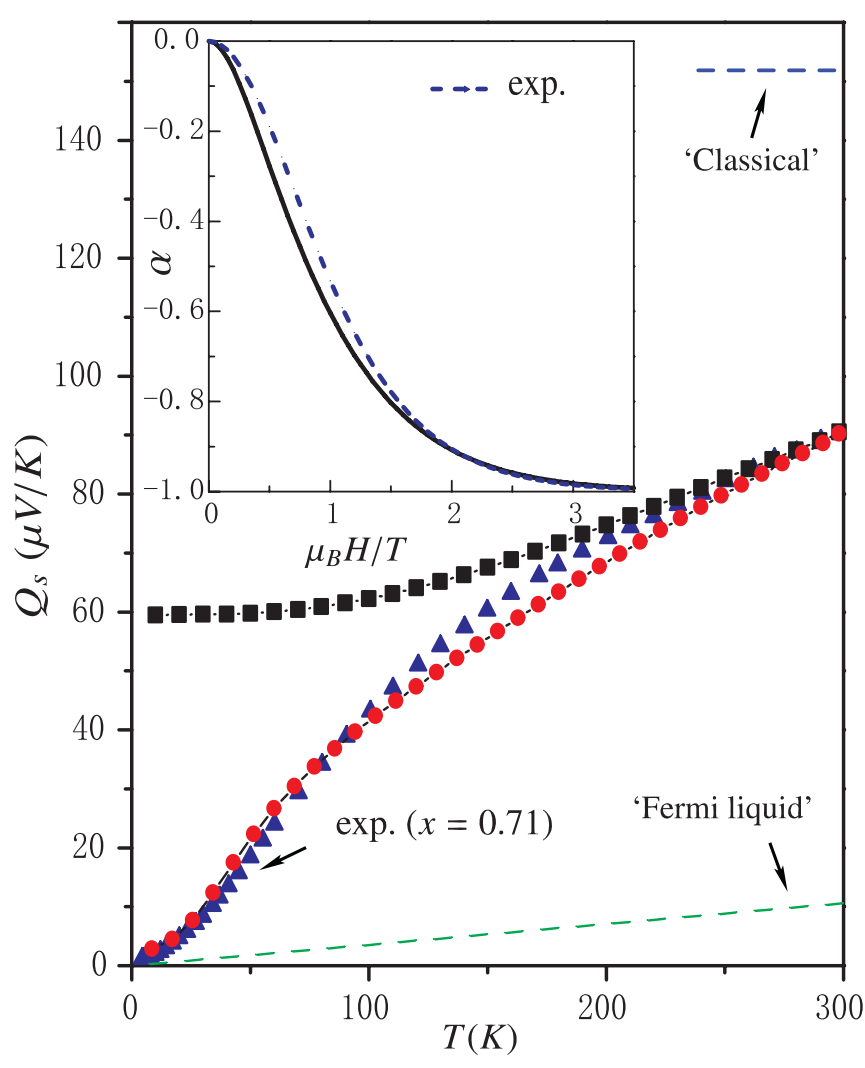

FIG. 2. (Color online) Thermopower $Q_{s}$ contributed by the spin part at $x=0.71$. Full squares: the mean-field solution; Full circles: the effect of doublon density fluctuations is considered (see text). The experimental result (full triangles) at the same $x$ as well as the Fermi liquid (dotted) and "classical limit" (dashed) results are shown for comparison. Inset: The scaling curve (solid) for $\alpha$ defined in Eq. (7) vs $H / T$ and the spin-entropy of free moments (dashed), which well accounts for the experiment measurement. ${ }^{6}$

where $t_{d}=-t B>0, t_{s}=-t D+J B / 4>0$. Here $a_{i j}$ represents the $U(1)$ gauge fluctuations around the saddle point: $\hat{D}_{i j} \simeq D e^{i a_{i j}}$ and $\hat{B}_{i j}^{\sigma} \simeq(B / 2) e^{i a_{i j}}$ which will recombine spins and charges together to electrons and destroy the mean-field saddle point. It can be shown ${ }^{21}$ that the saddle-point state is stable against the transverse fluctuations of $a_{i j}$, implying spin-charge separation. One simple way to see it is to note that the longitudinal component of $a_{i j}$ will reenforce the Mott constraint relaxed at the saddle-point level. Under such a constraint, the topological condition (4) may be replaced by $\sum_{\langle i j\rangle \in \partial S} A_{i j}^{d}=\pi \sum_{l \in S} \sum_{\sigma} n_{l \sigma}^{b}$, which means $H_{s}$ in (6) actually describes a two-component (with spin index $\sigma$ ) semionic system. (The spin index $\sigma$ before $A_{i j}^{d}$ actually guarantees the time reversal symmetry.) More detailed discussion will be presented elsewhere ${ }^{21}$ and in the following we shall focus on the saddle point at $a_{i j}=0$ in (5) and (6). As the phase string theory in the underdoped regime, this saddle point does not break any symmetries such as the time-reversal symmetry and spin rotation symmetry. ${ }^{4}$

Mean-field approximation. The spin dynamics is governed by $H_{s}$, where $A_{i j}^{d}$ given in Eq. (4) is only density dependent and can therefore, to leading order approximation, be treated 


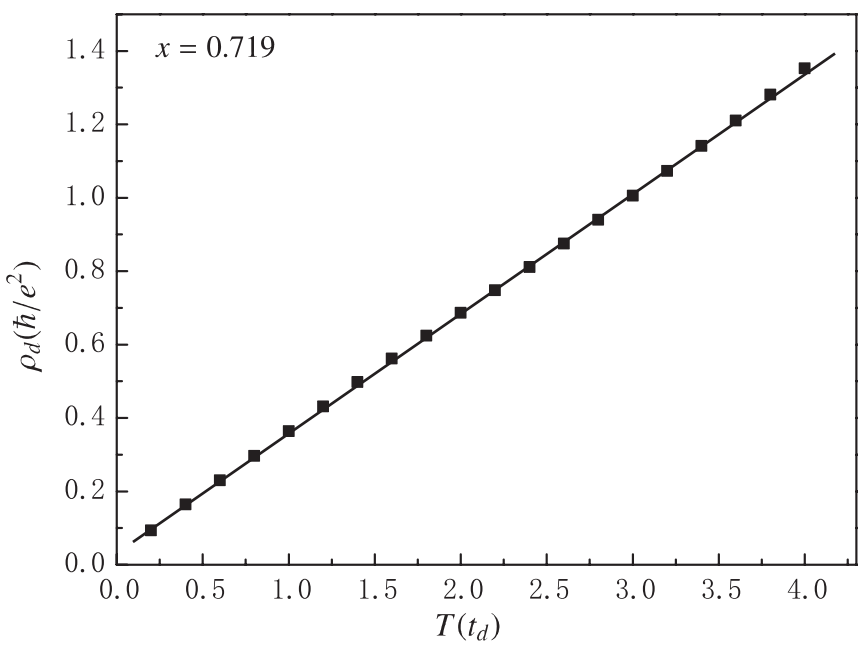

FIG. 3. The resistivity is contributed by doublons in the presence of random flux tubes $\pm \pi$ attached to spinons in the LLLs, which numerically treated as a random distribution of $\pm(1-\delta) \pi$ fluxes per unit cell.

as a smearing flux of $\pi(1-x)$ per unit cell. The resulting mean-field state of $H_{s}$ is Landau quantized with spins being statistically localized in the cyclotron orbits, in sharp contrast with a degenerate Fermi liquid state or a fully localized classical state at $t \rightarrow 0$. In particular, the characteristic bandwidth vanishes, while $t$ remains relatively large, when all the spinons stay in the degenerate LLLs at low temperatures, rendering a systematic scaling behavior as will be shown below.

Curie-Weiss uniform susceptibility. One peculiar property is exhibited by the uniform spin susceptibility, $\chi_{u}=\frac{2 \mu_{\mathrm{B}}^{2} \beta}{N} \sum_{m}\left(n_{m}+1\right) n_{m}$ where $\beta^{-1}=k_{\mathrm{B}} T$ and $n_{m}=$ $1 /\left[e^{\beta\left(E_{m}-\mu_{s}\right)}-1\right]$ is the Bose distribution for spinons at state $m$ with energy $E_{m}$ obtained by the aforementioned mean-field solution based on $H_{s}$ ( $\mu_{s}$ is the chemical potential). As clearly illustrated in Fig. 1 by $1 / \chi_{u}$, it follows a Curie-Weiss law $\chi_{u}=\frac{C}{T+\Theta}$ with $\Theta=0$ (full squares). A finite Weiss term $\Theta \simeq 3(1-x) J / k_{\mathrm{B}}$ (full circles) is generated by including an RPA correction from $H_{J}$ (by fitting with the experimental $\Theta{ }^{6}$ we estimate $J \simeq 70 \mathrm{~K}$ ). The coefficient $C$ is $x$ dependent, and at $T \rightarrow 0$ one finds $C=2(1-x) \mu_{\mathrm{B}}^{2} / k_{\mathrm{B}}$. As shown in the inset of Fig. 1, $C$ is in quantitative agreement with the experimental data $^{7,22,23}$ in the Curie-Weiss regime of the cobaltates, which is independent of other parameters in the model, like $t$ and $J$.

By contrast, a Pauli-like susceptibility is expected for the Fermi-liquid state (dotted line in Fig. 1, obtained with a bare $t=-0.1 \mathrm{eV}^{24}$ ). It is particularly instructive to compare this with the classical limit of the $t-J$ model at $t \rightarrow 0(J=0)$, where all the electrons are fully localized as $(1-x) N$ free moments, contributing to a Curie's law $\chi_{u}^{\mathrm{cl}}=C_{\mathrm{cl}} / T$ (dashed line in Fig. 1). But one finds $C_{\mathrm{cl}}=C / 2$, that is, only about the half of the values of both the experimental and the present theory (cf. the inset of Fig. 1). Clearly the peculiar quantum effect of the present bosonic spinons is responsible for the enhancement of $C$ from $C_{\mathrm{cl}}$ at low $T$ (only at high- $T$ limit, the "classical" $\chi_{u}^{\mathrm{cl}}$ can be recovered as $n_{m} \rightarrow 0$ in $\chi_{u}$ ).

Thermopower. In Fig. 2 the thermopowers predicted by the Fermi-liquid state as well as the classical limit of the $t-J$ model are shown by dotted and dashed curves, respectively. In

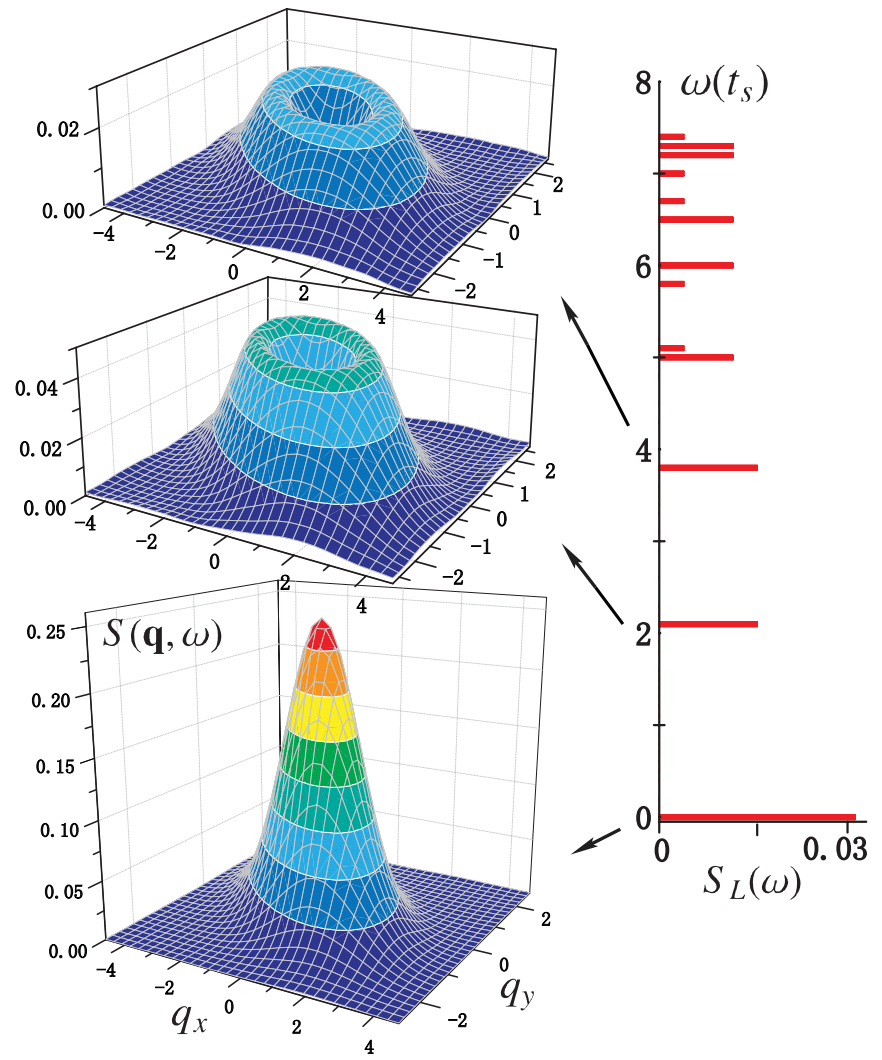

FIG. 4. (Color online) Dynamic spin structure factor $S(\mathbf{q}, \omega)$ predicted by the mean-field theory at $x=0.75$. Right panel: the Landau level structure exhibited in the local (q-integrated) dynamic structure factor $S_{L}(\omega)$. Left panel: the corresponding $\mathbf{q}$ dependence of $S(\mathbf{q}, \omega)$ for the first three energy levels.

the latter case, with the bandwidth vanishing, the thermopower reduces to the so-called Heikes formula: $Q_{\mathrm{cl}}=\left(k_{\mathrm{B}} / e\right) \ln \frac{2 x}{(1-x)}$ which is proportional to the entropy per electron. ${ }^{25,26}$ Both deviate strongly from the experimental result (full triangles in Fig. 2) in opposite ways.

In the present saddle-point state, the thermopower will satisfy the Ioffe-Larkin combination rule $Q=Q_{d}+Q_{s}$ according to Eqs. (5) and (6), with the spinon contribution $Q_{s}$ dominant over the doublon part $Q_{d}$. Here a large $Q_{s}$ originates in the degeneracy of the LLLs. This leads to a Heikes-like formula $Q_{s}=-\mu_{s} / e T$, associated with the spinon entropy, which follows given that the energy-particle current correlator $S^{12}=0$ at low temperature in the Kubo formula $Q_{s}=-\frac{1}{e T} \frac{S^{12}}{S^{11}}-\frac{\mu_{s}}{e T} \cdot{ }^{27}$ Note that the above Heikes-like formula is still valid even when $S^{12}$ becomes finite, for instance when the spinons are excited to the higher Landau levels at high $T$, because the current-current correlator $S^{11} \rightarrow \infty .^{21,28-30}$

The calculated $Q_{s}$ is presented in Fig. 2 (solid squares). This saturates at low $T$ to a universal value $Q_{s 0}=\frac{k_{\mathrm{B}}}{e} \ln 2 \approx$ $60 \mu \mathrm{V} / \mathrm{K}$ due to the artifact that the bandwidth of the LLLs remains zero. But a weak fluctuation of the gauge flux of $A_{i j}^{d}$ around the mean value can easily cause a broadening, lifting the exact LLL degeneracy, resulting in a vanishing $Q_{s}$ at $T \rightarrow 0$. A typical example is indicated by the full circles in Fig. 2, with $A_{i j}^{d}$ simulated by a random flux of $[-0.3 \pi, 0.3 \pi]$ 
around the mean flux of $\pi(1-x)$ per unit cell. One finds a quantitative agreement with the experimental measurement by taking $t_{s}=100 \mathrm{~K}$. Note that $Q_{d}$ contributed by doublons is not included here as it is usually much weaker in magnitude with a quite flat $T$ dependent as calculated by the Kubo formula. ${ }^{21}$

To understand more clearly the origin of $Q_{s}$ from the spin degrees of freedom, one may apply a strong in-plane magnetic field $H$ to polarize (freeze) the spins via Zeeman energy, as already accomplished experimentally. ${ }^{6}$ Denote $Q_{s}(\infty)$ as the contribution from the remaining configurational entropy at $H \rightarrow \infty$ and define

$$
\alpha=\frac{Q_{s}(H)-Q_{s}(\infty)}{Q_{s}(0)-Q_{s}(\infty)}-1
$$

Then $\alpha$ is found to be a well-defined scaling curve of $H / T$, as shown by the solid curve in the inset of Fig. 2, which fits well with the pure spin entropy of free moments: $[\ln [2 \cosh (u)]-u \tanh (u)] / \ln 2$, where $u=$ $\left(2.2 \mu_{\mathrm{B}} H\right) /\left(2 k_{\mathrm{B}} T\right)$ in excellent agreement with the experimental data as given in Ref. 6 .

Resistivity. The resistivity satisfies the Ioffe-Larkin combination rule $\rho=\rho_{d}+\rho_{s}$ in which $\rho_{s}=0$ due to the aforementioned "Meissner effect" response $\mathrm{e}^{21,28-30}$ in the spinon subsystem such that the doublon subsystem will solely contribute to $\rho$. Governed by $H_{d}$, the doublons will experience the novel scattering generated from the gauge potential $A_{i j}^{s}$, which describes $\pm \pi$ flux tubes bound to the spinons with $\sigma= \pm$. In the low- $T$ regime where the spinons are in the degenerate Landau orbits, the corresponding flux tubes seen by the holons will distribute randomly in space. Based on a numerical calculation where $A_{i j}^{s}$ is treated as randomly distributed $\pm(1-x) \pi$ fluxes per unit cell, we obtain a metallic behavior of $\rho_{d}$ with a linear- $T$ dependence over a large temperature regime as shown in Fig. 3, originating in a scattering rate similar to a case previously studied ${ }^{31}$ in a high- $T$ regime in the context of high- $T_{c}$ cuprates.

Predictions and discussion. The fractionalized saddle-point state governed by (5) and (6) has been shown to generically exhibit a systematic scaling behavior: $\rho \propto T, \chi_{u} \propto 1 / T$, a large thermopower $Q_{s} \sim \frac{k_{\mathrm{B}}}{e} \ln 2$, as well as the scaling law in $\alpha=\alpha(H / T)$, associated with the peculiar Landau quantization effect in $H_{s}$. Our theory allows us to make one further strong prediction: the spin sector Landau levels can be in fact directly observed by inelastic neutron scattering! The dynamic spin structure factor $S(\mathbf{q}, \omega)$ can be easily computed assuming the straightforward Landau quantization and it should look like Fig. 4: a tower of rather narrow nondispersive bands of spin fluctuation (right panel), with a momentum dependence as indicated in the left panel.

Another interesting consequence of the spin-charge separation is in the figure of merit used to quantify the efficiency of a thermoelectric device. This can be written in the following form:

$$
Z T \equiv \frac{Q^{2} T}{\rho \kappa}=\frac{\left(Q_{s}+Q_{d}\right)^{2} / L_{d}}{1+\kappa_{p h} / \kappa_{d}}
$$

since the spinons do not contribute to the thermal conductivity and resistivity $\left(\kappa_{s}=0\right.$ and $\left.\rho_{s}=0\right) .^{21}$ Then a large spinon thermopower $Q_{s}\left(\gg\left|Q_{d}\right|\right)$ will play an important role, independent of the Lorenz number of the doublons $\mathfrak{七}_{d} \equiv$ $\frac{T}{\kappa_{d} \rho_{d}}$ as well as the doublon $\left(\kappa_{d}\right)$ and phonon $\left(\kappa_{p h}\right)$ thermal conductivities. We find ${ }^{21} L_{d}$ to be one order of magnitude smaller than the Lorenz number of a Fermi liquid. This is in fact consistent with exact diagonalization result on the $t-J$ model. ${ }^{32}$ The combination of these factors may therefore lead potentially to an exceptionally large $Z$.

Finally, we emphasize that in principle a sharp LLL degeneracy is an artifact of the mean-field approximation which can be easily lifted, for instance, by density fluctuations via $A_{i j}^{d}$ intrinsically or extrinsically (e.g., induced by the distribution of $\mathrm{Na}),{ }^{33-35}$ which leads to other stable phases at low temperature. Nonetheless, the mean-field results is expected to remain a good approximation to the saddle-point physics at "intermediate" temperatures higher than the LLL broadening. Namely, the physics we have discussed here is associated with an intermediate temperature regime, bounded by the Landau gap scale at high temperature and the onset of further cooperative phenomena at low temperatures. In the latter regime the specific heat measurements have also shown $^{36,37}$ an anomalous increase, presumably due to the afore-discussed entropy enhancement which, however, will be sensitive to the detailed Landau level broadening. Eventually the spin degeneracies associated with the Landau states will be lifted, and this might be the origin of some new emergent orders at very low temperatures. One infers from the momentum dependence of $S(\mathbf{q}, \omega)$ at the LLLs (Fig. 4) that the in-plane ferromagnetic correlation length will increase with $x$, such that a small interlayer superexchange $J_{\perp}$ may eventually drive an in-plane ferromagnetic order with antiferromagnetic ordering along different layers, explaining the $A$-type antiferromagnetic order observed by neutron scattering at $x \geqslant 3 / 4 .{ }^{38}$ The bosonic doublons may also condense at a sufficiently low $T$ to make the system more Fermi-liquid like, reconciling with the Wiedemann-Franz law observed in $\mathrm{Na}_{0.7} \mathrm{CoO}_{2}$ at $T<1 \mathrm{~K}$. ${ }^{39}$ However our unconventional "building material" also leaves room for less conventional forms of order, including uncommon charge orders that will be discussed in detail elsewhere.

We acknowledge stimulating discussions with N. P. Ong, X. L. Qi, and in particular Y. Wang who also provided us with unpublished experimental data. This work was supported by the grants of NSFC and the National Program for Basic Research of MOST, China, and a Spinoza grant of the Nederlandse Organisatie voor Wetenschappelijk Onderzoek (NWO).
${ }^{1} \mathrm{P}$. W. Anderson, The Theory of Superconductivity in the High $T_{c}$ Cuprates (Princeton University Press, Princeton, 1997).

${ }^{2}$ J. Zaanen and B. J. Overbosch, e-print arXiv:0911.0470.
${ }^{3}$ P. W. Anderson, Science 235, 1196 (1987).

${ }^{4}$ Z.-Y. Weng, Int. J. Mod. Phys. B 21, 773 (2007). 
${ }^{5}$ I. Terasaki, Y. Sasago, and K. Uchinokura, Phys. Rev. B 56, R12685 (1997).

${ }^{6}$ Y. Wang et al., Nature (London) 423, 425 (2003).

${ }^{7}$ M. L. Foo, Y. Wang, S. Watauchi, H. W. Zandbergen, T. He, R. J. Cava, and N. P. Ong, Phys. Rev. Lett. 92, 247001 (2004).

${ }^{8}$ M. Lee et al., Nat. Mater. 5, 537 (2006).

${ }^{9}$ O. I. Motrunich and P. A. Lee, Phys. Rev. B 69, 214516 (2004).

${ }^{10}$ J. O. Haerter, M. R. Peterson, and B. S. Shastry, Phys. Rev. Lett. 97, 226402 (2006).

${ }^{11}$ K. Kuroki and R. Arita, J. Phys. Soc. Jpn. 76, 083707 (2007).

${ }^{12}$ C. A. Marianetti and G. Kotliar, Phys. Rev. Lett. 98, 176405 (2007).

${ }^{13}$ F. Lechermann, Phys. Rev. Lett. 102, 046403 (2009).

${ }^{14}$ G. Khaliullin and J. Chaloupka, Phys. Rev. B 77, 104532 (2008).

${ }^{15}$ G. Baskaran, Phys. Rev. Lett. 91, 097003 (2003).

${ }^{16}$ B. Kumar and B. S. Shastry, Phys. Rev. B 68, 104508 (2003).

${ }^{17}$ Q.-H. Wang, D.-H. Lee, and P. A. Lee, Phys. Rev. B 69, 092504 (2004).

${ }^{18}$ M. Z. Hasan et al., Phys. Rev. Lett. 92, 246402 (2004).

${ }^{19}$ D. Qian, L. Wray, D. Hsieh, L. Viciu, R. J. Cava, J. L. Luo, D. Wu, N. L. Wang, and M. Z. Hasan, Phys. Rev. Lett. 97, 186405 (2006). ${ }^{20}$ S. Zhou, M. Gao, H. Ding, P. A. Lee, and Z. Wang, Phys. Rev. Lett. 94, 206401 (2005).

${ }^{21} \mathrm{~K}$. Wu, Z. Y. Weng, and J. Zaanen (unpublished).

${ }^{22}$ R. Ray, A. Ghoshray, K. Ghoshray, and S. Nakamura, Phys. Rev. B 59, 9454 (1999).
${ }^{23}$ T. Motohashi, R. Ueda, E. Naujalis, T. Tojo, I. Terasaki, T. Atake, M. Karppinen, and H. Yamauchi, Phys. Rev. B 67, 064406 (2003).

${ }^{24}$ D. J. Singh, Phys. Rev. B 60, 13397 (2000).

${ }^{25}$ G. Beni, Phys. Rev. B 10, 2186 (1974).

${ }^{26}$ P. M. Chaikin and G. Beni, Phys. Rev. B 13, 647 (1976).

${ }^{27}$ Here $\mu_{s}$ is defined with taking the LLL energy $E_{0}=0$ such that $\mu_{s}(T=0)=0$.

${ }^{28}$ R. B. Laughlin, Phys. Rev. Lett 60, 2677 (1988).

${ }^{29}$ A. L. Fetter, C. B. Hanna, and R. B. Laughlin, Phys. Rev. B 39, 9679 (1989).

${ }^{30}$ Y H. Chen, F. Wilczek, E. Witten, and B. I. Halperin, Int. J. Mod. Phys. B 3, 1001 (1989).

${ }^{31}$ Z.-C. Gu and Z.-Y. Weng, Phys. Rev. B 76, 024501 (2007).

${ }^{32}$ M. R. Peterson, B. S. Shastry, and J. O. Haerter, Phys. Rev. B 76, 165118 (2007).

${ }^{33}$ I. R. Mukhamedshin, H. Alloul, G. Collin, and N. Blanchard, Phys. Rev. Lett. 93, 167601 (2004).

${ }^{34}$ H. Alloul et al., Europhys. Lett. 82, 17002 (2008).

${ }^{35} \mathrm{H}$. Alloul et al., Europhys. Lett. 85, 47006 (2009).

${ }^{36}$ T. F. Schulze et al., Phys. Rev. B 78, 205101 (2008).

${ }^{37}$ Y. Okamoto, A. Nishio, and Z. Hiroi, Phys. Rev. B 81, 121102 (2010).

${ }^{38}$ A. T. Boothroyd, R. Coldea, D. A. Tennant, D. Prabhakaran, L. M. Helme, and C. D. Frost, Phys. Rev. Lett. 92, 197201 (2004).

${ }^{39}$ Y.-S. Li et al., Phys. Rev. Lett. 93, 056401 (2004). 